\title{
Decreased blood natural killer cells in serofast patients: a systematic review and meta-analysis
}

\author{
WENHAO CHENG, YUMO LU, RENQIONG CHEN, HONG REN, WENLONG HU
}

The Affiliated Lianyungang Hospital of Xuzhou Medical University, China

\begin{abstract}
Currently, increasing attention has been paid to the association of the serofast status with natural killer (NK) cells. Remarkable diversity among the results of different studies has been observed. We conducted this meta-analysis to evaluate the variation of the proportion of NK cells in serofast patients compared with that of healthy controls and cured patients. Through the designed retrieval methods, 631 serofast patients, 562 healthy controls and 160 patients whose serology turned negative following treatment were derived from 16 publications for further analysis. The established items were used for the standard selection and quality assessment. The Stata software was used for meta-analysis. The final results indicated that serofast patients exhibited a dramatic decrease in the number of NK cells in the peripheral blood compared with that noted in healthy control subjects [standardized mean difference $(S M D)=-0.63,95 \% C I(-1.08,-0.17), p=0.007]$. The proportion of NK cells was significantly lower in serofast patients than that noted in cured patients [SMD $=-0.25,95 \%$ CI $(-0.48,-0.02)$, $p=0.033$ ] and no significant difference was noted in the proportion of $N K$ cells between cured patients and healthy controls [SMD $=-0.39,95 \% C I(-0.93,0.14), p=0.148]$. The present meta-analysis indicated that the proportion of $\mathrm{NK}$ cells in the peripheral blood was significantly lower in serofast patients compared with that of the healthy controls and cured patients, indicating that the reduction in the number of NK cells may be closely associated with the syphilis serofast status.
\end{abstract}

Key words: meta-analysis, syphilis, natural killer cells, serofast.

(Cent Eur J Immunol 2021; 46 (4): 509-515)

\section{Introduction}

Syphilis is a chronic infectious transmitted disease caused by Treponema pallidum (TP) with various clinical manifestations. It occurs widely all over the world and poses a challenge to global public health. Concomitantly, this disease increases the risk of human immunodeficiency virus (HIV) infection and transmission [1,2]. Syphilis not only leads to the development of skin mucosal lesions, but also causes irreversible damage to the cardiovascular and central nervous systems. In addition, untreated pregnant women with active syphilis can suffer from miscarriage, stillbirth, premature delivery and fetal syphilis [3]. More than 12 million newly diagnosed syphilis cases have been reported worldwide each year [1]. Moreover, the rising incidence of syphilis in recent years has been accompanied with a significant increase in the ratio of syphilis serofast $[4,5]$.

At present, there is no uniform regulation on syphilis serofast in the world. Chinese researchers produced a relatively uniform definition as a reference for this condition in 2015 [6]. The following criteria are used: syphilis patients must have undergone standard anti-syphilis treatment and full follow-up (the follow-up time for primary syphilis, secondary syphilis and latent syphilis is 1 year, 2 and 3 years respectively), and non-treponemal serological tests must have been maintained at a certain titer (generally $\leq 1: 8$, but $>1: 8$ is not uncommon) for more than 3 months, excluding biological false positives, reinfection, neurosyphilis and cardiovascular syphilis. This is defined as syphilis serofast by the Centers of Disease Control and Prevention (CDC) and represents the lack of at least a 4-fold decline in the titer of the non-treponemal assay in the period between 6 and 12 months following treatment in the absence of progressive disease and in spite of almost complete cure success [7].

Although the specific mechanism of serofast in syphilis patients is not clear, the majority of the studies have shown that the immune function of syphilis serofast patients has changed [8-11]. Natural killer (NK) cells are the major members of the innate immune system and exert anti-infective effects via the production of various cytokines and cytotoxic effectors [12]. Several studies have been performed with regard to the number of NK cells in serofast patients and have produced distinct results. A significant association between NK cells and serofast patients

Correspondence: Wenlong Hu, The Affiliated Lianyungang Hospital of Xuzhou Medical University, China, e-mail: lyghuwl@163.com Submitted: 21.03.2020; Accepted: 2.07.2021 
was observed in certain studies, while other reports did not produce consistent results $[13,14]$. Therefore, the objective of this meta-analysis was to systematically evaluate the variation of the proportion of NK cells in serofast patients compared with that of healthy control subjects and cured patients.

\section{Material and methods}

\section{Study selection}

The case-control studies were identified by searching PubMed, Cochrane Library, Google Scholar, China National Knowledge Infrastructure (CNKI), Wan Fang database and the Chinese and Scientific Journals Full text database (CQVIP) from database inception to October 2019. The combination of medical subject heading terms used was as follows: (serofast OR seroresistance) AND (natural killer cells OR NK cells). No language restrictions were applied to the search. The complete citation screening process was based on the Preferred Reporting Items for Systematic Reviews and Meta-Analyses Statement (PRISMA) [15].

\section{Inclusion and exclusion criteria}

The inclusion criteria for this investigation included case-control studies that measured NK cells in the sera of serofast patients and compared them with control subjects. Initially, duplicate studies and studies that lacked important data were eliminated. Case reports, reviews, letters, conference abstracts and meta-analyses were excluded as well.

\section{Data extraction}

We extracted the following data from each of the included studies: study design, publication year, first author, sample composition, sample size and outcome. All data associated with NK cells were also extracted for further statistical analysis. The researchers extracted data from the included articles and an additional researcher finally examined the accuracy of the extracted data. We also contacted the author if important data were unclear or questionable.

\section{Statistical analysis}

STATA 15.0 was used for the present meta-analysis. We used the $I^{2}$ statistical test to evaluate heterogeneity. The degree of heterogeneity was considered small or negligible if $I^{2} \leq 50 \%$. Whenever $I^{2}>50 \%$, the heterogeneity was considered significant and the random-effects model was used. For all the outcomes, the data with a $p$-value lower than 0.05 were considered significant. The standardized mean difference (SMD) with $95 \%$ confidence interval $(95 \% \mathrm{CI})$ was used to describe the continuous data. The association between the proportion of NK cells and serofast was assessed by these values and the results were presented as forest plots. Finally, the publication bias test was carried out on the included literature by Begg's test $(\alpha=0.05)$.

\section{Results}

\section{Literature retrieval}

A total of 265 Chinese and English studies were retrieved using the retrieval strategy. Two staff members, in strict accordance with the inclusion and exclusion criteria of the study design, performed preliminary screening, fine screening and final screening and finally 16 studies were included. The main characteristics of the studies included are shown in Table 1.

\section{Meta-analysis}

The comparison of the percentage of NK cells in the peripheral blood of patients in the serofast group was made with that of the healthy control group. The analysis included 16 articles [14, 16-30], 631 patients in the serofast group and 562 patients in the healthy control group. Statistical heterogeneity between the results of each study was noted $\left(I^{2}=92.6 \%\right)$. However, when no clinical heterogeneity was present, the random-effects model was used for data combination analysis. The mean value of each study was considerably different and SMD was adopted as the effect value. The combined results indicated that the proportion of NK cells in serofast patients was significantly lower compared with that of the healthy control subjects. The differences noted were significant $[\mathrm{SMD}=-0.63$, 95\% CI (-1.08, -0.17), $p=0.007$ ] (Fig. 1).

In addition, the proportion of NK cells in serofast patients $(n=138)$, healthy control subjects $(n=148)$ and cured patients $(n=160)$ was compared. The data were extracted from four studies [14, 16-19]. The results revealed that the proportion of NK cells was significantly lower in serofast patients than that noted in cured patients [SMD $=-0.25,95 \%$ CI $(-0.48,-0.02), p=0.033$ ] (Fig. 2). Due to the decreased heterogeneity $\left(I^{2}=0.0 \%\right)$, the fixed-effects model was applied. In addition, no significant differences were noted with regard to the proportion of NK cells among cured patients and healthy subjects [SMD = $-0.39,95 \%$ CI $(-0.93,0.14), p=0.148$ ] (Fig. 3). Due to the apparent heterogeneity $\left(I^{2}=80.6 \%\right)$, the random-effects model was used. The summary of the meta-analysis on NK cells in serofast patients, healthy controls and cured patients is shown in Table 2.

\section{Publication bias}

Begg's test was performed to assess the potential publication bias of all comparisons and the results indicated the absence of publication bias (Table 2). Funnel plots revealed the absence of asymmetry as expected. 
Table 1. Main characteristics of the studies included in the meta-analysis

\begin{tabular}{|c|c|c|c|c|c|c|c|c|c|c|}
\hline \multirow[t]{2}{*}{ Study } & \multirow[t]{2}{*}{ Year } & \multicolumn{3}{|c|}{ Serofast patients } & \multicolumn{3}{|c|}{ Healthy controls } & \multicolumn{3}{|c|}{ Cured patients } \\
\hline & & $n$ & Mean & SD & $n$ & Mean & SD & $n$ & Mean & SD \\
\hline Huang & 2018 & 103 & 8.10 & 0.80 & 103 & 8.70 & 0.80 & - & - & - \\
\hline Xie & 2017 & 33 & 7.32 & 4.48 & 23 & 14.87 & 6.26 & - & - & - \\
\hline Liang & 2016 & 60 & 21.58 & 6.11 & 60 & 14.18 & 4.55 & - & - & - \\
\hline Liu & 2015 & 35 & 15.10 & 4.20 & 40 & 20.60 & 5.20 & 40 & 16.50 & 4.40 \\
\hline Xiong & 2015 & 60 & 17.96 & 7.67 & 60 & 22.35 & 7.85 & - & - & - \\
\hline $\mathrm{Xu}$ & 2014 & 40 & 8.20 & 0.70 & 40 & 8.60 & 0.60 & 50 & 8.50 & 0.70 \\
\hline Xie & 2013 & 32 & 15.03 & 4.98 & 11 & 22.25 & 6.11 & - & - & - \\
\hline Zhang & 2013 & 24 & 14.91 & 6.98 & 22 & 20.10 & 9.01 & - & - & - \\
\hline Guan & 2013 & 54 & 18.39 & 7.93 & 32 & 22.84 & 8.47 & - & - & - \\
\hline Ruan & 2012 & 32 & 14.91 & 6.98 & 30 & 20.10 & 9.01 & - & - & - \\
\hline Lin & 2010 & 23 & 15.82 & 8.44 & 20 & 20.35 & 9.93 & 20 & 16.81 & 6.95 \\
\hline Liu & 2010 & 25 & 10.41 & 7.21 & 20 & 25.67 & 7.76 & - & - & - \\
\hline $\mathrm{Li}$ & 2009 & 20 & 13.00 & 6.00 & 18 & 16.00 & 6.00 & 20 & 13.00 & 8.00 \\
\hline Huang & 2008 & 20 & 20.55 & 6.55 & 30 & 18.90 & 4.84 & 30 & 21.27 & 4.98 \\
\hline Bao & 2006 & 38 & 0.06 & 0.02 & 23 & 0.13 & 0.04 & - & - & - \\
\hline Yang & 2005 & 32 & 21.59 & 7.32 & 30 & 14.19 & 4.87 & - & - & - \\
\hline
\end{tabular}

\begin{tabular}{|c|c|c|}
\hline $\begin{array}{l}\text { Study } \\
\text { ID }\end{array}$ & $\operatorname{SMD}(95 \% \mathrm{CI})$ & $\begin{array}{c}\% \\
\text { Weight }\end{array}$ \\
\hline Huang (2018) & $-0.75(-1.03,-0.47)$ & 6.70 \\
\hline Xie (2017) & $-1.43(-2.03,-0.83)$ & 6.15 \\
\hline Liang (2016) & $1.37(0.98,1.77)$ & 6.53 \\
\hline Liu (2015) & $-1.16(-1.65,-0.66)$ & 6.37 \\
\hline Xiong (2015) & $-0.57(-0.93,-0.20)$ & 6.58 \\
\hline $\mathrm{Xu}(2014)$ & $-0.61(-1.06,-0.16)$ & 6.45 \\
\hline Xie (2013) & $-1.37(-2.11,-0.62)$ & 5.81 \\
\hline Zhang (2013) & $-0.65(-1.24,-0.05)$ & 6.16 \\
\hline Guan (2013) & $-0.55(-0.99,-0.10)$ & 6.45 \\
\hline Ruan (2012) & $-0.65(-1.16,-0.14)$ & 6.33 \\
\hline $\operatorname{Lin}(2010)$ & $-0.49(-1.10,0.11)$ & 6.13 \\
\hline Liu (2010) & $-2.05(-2.78,-1.32)$ & 5.85 \\
\hline Li (2009) & $-0.50(-1.15,0.1 w 5)$ & 6.04 \\
\hline Huang (2008) & $0.30(-0.27,0.86)$ & 6.21 \\
\hline Bao (2006) & $-2.40(-3.08,-1.73)$ & 5.98 \\
\hline Yang (2005) & $1.18(0.64,1.72)$ & 6.27 \\
\hline Overall $\left(I^{2}=92.6 \%, p=0.000\right)$ & $-0.63(-1.08,-0.17)$ & 100.00 \\
\hline Note. Weights are from random effects analysis. & & \\
\hline-5 & 5 & \\
\hline
\end{tabular}

Fig. 1. Forest plot of the proportion of NK cells in serofast patients and healthy controls 


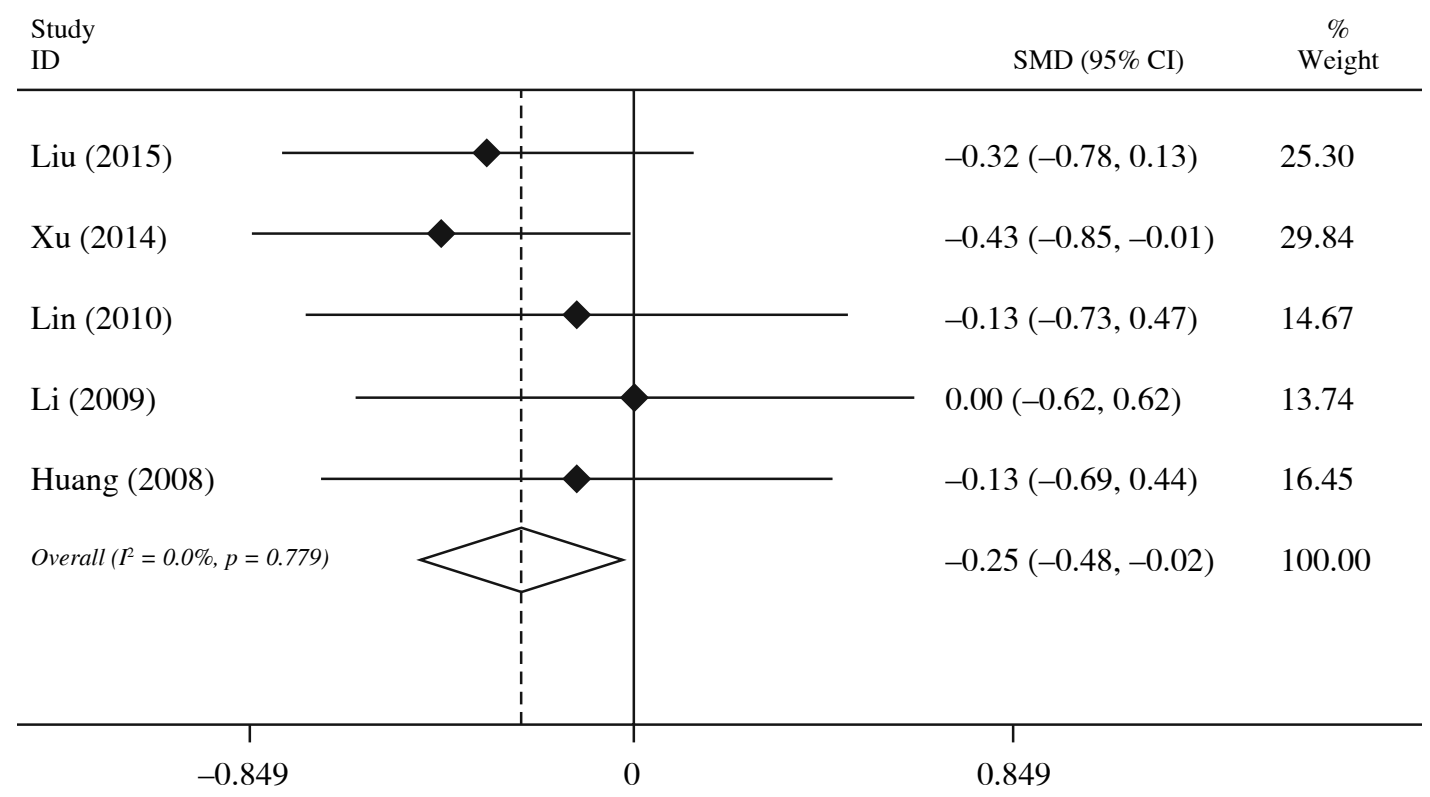

Fig. 2. Forest plot of the proportion of NK cells in serofast patients and cured patients

\begin{tabular}{|c|c|c|}
\hline $\begin{array}{l}\text { Study } \\
\text { ID }\end{array}$ & SMD $(95 \%$ CI) & $\begin{array}{c}\% \\
\text { Weight }\end{array}$ \\
\hline Liu (2015) & $-0.85(-1.31,-0.39)$ & 21.25 \\
\hline Xu (2014) & $-0.15(-0.57,0.26)$ & 21.84 \\
\hline Lin (2010) & $-1.11(-1.78,-0.44)$ & 18.06 \\
\hline $\mathrm{Li}(2009)$ 0.22) & $-0.42(-1.07,0.22)$ & 18.43 \\
\hline Huang (2008) & $-0.48(-0.03,1.00)$ & 20.43 \\
\hline Overall $\left(I^{2}=80.6 \%, p=0.000\right)$ & $-0.39(-0.93,0.14)$ & 100.00 \\
\hline Note. Weights are from random effects analysis. & & \\
\hline$T$ & & $T$ \\
\hline-5 & & 5 \\
\hline
\end{tabular}

Fig. 3. Forest plot of the proportion of NK cells in cured patients and healthy controls

\section{Discussion}

NK cells are core members of the natural immune system. The activity of NK cells determines the immune function of the body to some extent. With regard to prevention of infection, NK cells not only kill cells infected by pathogens by direct contact with them, but also secrete cytokines to control the proliferation of pathogens in the cells [31]. A previous study showed that NK cells rapidly secrete a large number of cytokines following activation and mobilize NK cells to participate in the activation process. Furthermore, the speed and intensity of activation are higher than those of the T cells [32]. During infection, the activity of NK cells is regulated by the interaction of stimulatory and inhibitory signals on the cell surfaces. NK cells play an immune killing function in the early stage of pathogen infection. Furthermore, NK cells can also release cytokines for immune regulation and serve as a bridge connecting adaptive and innate immunity. NK cells play an irreplaceable role in the prevention of infection. A large 
Table 2. Summary of the meta-analysis on NK cells: serofast patients, healthy controls and cured patients

\begin{tabular}{|c|c|c|c|c|c|c|c|c|}
\hline \multirow[t]{2}{*}{ Comparisons } & \multirow{2}{*}{$\begin{array}{l}\text { No. of } \\
\text { compa- } \\
\text { risons }\end{array}$} & \multicolumn{3}{|c|}{ Test of association } & \multicolumn{3}{|c|}{ Test of heterogeneity } & \multirow{2}{*}{$\begin{array}{c}\text { Publication bias } \\
\begin{array}{c}p \text {-value } \\
\text { (Begg's test) }\end{array}\end{array}$} \\
\hline & & SMD & $95 \%$ CI & $p$-value & Model & $I^{2}(\%)$ & $p$-value & \\
\hline $\begin{array}{l}\text { Serofast patients } \\
\text { vs. healthy controls }\end{array}$ & 16 & -0.63 & $\begin{array}{c}-1.08 \sim- \\
0.17\end{array}$ & 0.007 & $\mathrm{R}$ & 92.6 & 0.000 & 0.192 \\
\hline $\begin{array}{l}\text { Serofast patients } \\
\text { vs. cured patients }\end{array}$ & 5 & -0.25 & $\begin{array}{c}-0.48 \sim- \\
0.02\end{array}$ & 0.033 & $\mathrm{~F}$ & 0.0 & 0.779 & 0.806 \\
\hline $\begin{array}{l}\text { Cured patients } \\
\text { vs. healthy controls }\end{array}$ & 5 & -0.39 & $-0.93 \sim 0.14$ & 0.148 & $\mathrm{R}$ & 80.6 & 0.000 & 0.806 \\
\hline
\end{tabular}

$R$-random-effects model, $F$ - fixed-effects model

number of studies have also shown that the lack of NK cells is closely associated with fatal complications caused by various serious infections.

Recent studies have made significant progress in the mechanism of NK cells in immune or infectious diseases. However, previous work has focused on the function of NK cells in identifying and responding to bacteria, viruses, parasitic infections and tumors [12,33]. A limited number of reports have examined the role of NK cells in TP infection. A previous study showed that a high number of NK cells that infiltrated the TP infected skin were present in addition to CD4+ and CD8+ T cells, whereas the number of NK cells in peripheral blood was significantly reduced in secondary syphilis and was accompanied by the presence of immature NK cell subtypes [34]. This subset of NK cells exhibited a poor ability to lyse the cells and a low ability to produce interferon $\gamma($ IFN- $\gamma$ ) and other cytokines [35]. We envisage that NK cells play an important role in the immune escape of TP and the formation of serofast.

Numerous studies have shown that the number of NK cells present in the peripheral blood of the serofast group is significantly lower than that of the healthy subjects [16-20, 23-30]. However, certain studies have documented conflicting results [21,22], and it has been shown that NK cells comprise an antibody-dependent cell-mediated cytotoxicity (ADCC) effector type of cells, which can only function by the combination of the antibodies and the target cells. The number of TP bacteria in serum-fixed patients was significantly reduced following the appropriate treatment. The immune function of NK cells is weakened and certain TP antigens remain in the body to form serofast. Currently, there is a lack of consensus on the changes in the number of NK cells in serofast peripheral blood specimens.

The present study is the first meta-analysis to perform an extensive and comprehensive assessment of the association between NK cells and serofast. It can provide certain evidence-based results for a controversial issue based on current publications. We extracted the results from several studies and reduced the potential influence of the small sample size of the results by increasing the sample size. The analysis indicated that the proportion of NK cells in the serofast syphilis group was significantly lower than that of the healthy control and cured patient groups. The results did not indicate a significant difference in the proportion of NK cells between the cured patients and healthy control group.

The levels of cytokines secreted by NK cells determine the immune status of the body to a certain extent. Following activation, NK cells participate in the activation of macrophages by secreting IFN- $\gamma$ and by maintaining the dominant state of Th1 in order to determine the differentiation of cytotoxic lymphocyte precursor cells into mature cytotoxic lymphocytes [12, 32]. A previous study showed that macrophages can engulf and clear TP antigens, while they can also lower the number of TP bacteria at the infection site via ADCC [36]. Based on the results of our meta-analysis, the proportion of NK cells in the peripheral blood of serofast patients was significantly decreased, whereas the ability of NK cells to secrete IFN- $\gamma$ was weakened. Therefore, we speculated that TP infection could affect the number of NK cells and reduce their immune function by indirectly affecting the immune functions of macrophages and Th1 cells [37]. Therefore, NK cells cannot completely prevent the invasion of $\mathrm{TP}$, which remains in a state of continuous infection in the human body, leading to the formation of serofast.

The current study has several limitations. Firstly, although we conducted a methodological evaluation to avoid selection bias, the results of the heterogeneity analysis indicated significant heterogeneity among various studies, which may lead to incomparability of the research results. Fortunately, no clinical heterogeneity was evident among the different studies and therefore the random-effects model was adopted to merge the SMD of similar studies, control their heterogeneity and ensure the reliability of the results. Secondly, the data were interpreted cautiously due to the small number of published studies available on this topic and most studies used small sample sizes. Therefore, the current study emphasizes the necessity of conducting additional trials to evaluate the association between NK cells and serofast. Thirdly, no uniform diagnostic criteria were reported for serofast, suggesting that the appropriate methodology to assess the serological response is not 
known. Therefore, the random misclassification of serofast may affect the outcome. Fourthly, significant heterogeneity was noted in the present study. Moreover, we could not determine the source of heterogeneity by sensitivity and subgroup analyses. Finally, since the current results were mainly based on data from the Chinese population, further research is required on other populations to verify these findings.

In conclusion, the present meta-analysis indicated that the proportion of NK cells in peripheral blood was significantly lower in serofast patients compared with that in healthy subjects and cured patients, indicating that the reduction of NK cells may be closely associated with syphilis serofast. However, the exact role of the decreased number of blood NK cells in the formation of serofast is still not completely clear. Further studies on the association between NK cells and the pathogenesis of serofast may provide a basis for the prevention and treatment of this condition in the future.

\section{Acknowledgments}

This work was supported by grants from the National Natural Science Foundation of China (grant numbers: 81602769).

\section{The authors declare no conflict of interest.}

\section{References}

1. Kojima N, Klausner JD (2018): An update on the global epidemiology of syphilis. Curr Epidemiol Rep 5: 24-38.

2. Wankwo A, Okuonghae D (2018): Mathematical analysis of the transmission dynamics of HIV syphilis co-infection in the presence of treatment for syphilis. Bull Math Biol 80: 437-492.

3. Curry SJ, Krist AH, Owens DK, et al. (2018): Screening for syphilis infection in pregnant women: US preventive services task force reaffirmation recommendation statement. JAMA 32: 911-917.

4. Stamm LV (2016): Syphilis: Re-emergence of an old foe. Microbial cell (Graz, Austria) 3: 363-370.

5. Kenyon CR, Osbak KK, Van Esbroek M, et al. (2018): What is the role of paired rapid plasma reagin testing (simultaneous testing of acute and convalescent samples) in the diagnosis of repeat syphilis and the follow-up of syphilis? Sex Transm Dis 45: 35-38.

6. Wang QQ, Fan RQ (2015): Expert agreement on clinical treatment of syphilis serofast. Chin J Dermatol 48: 753-754.

7. Workowski KA, Bolan GA (2015): Sexually transmitted diseases treatment guidelines, 2015. MMWR Recomm Rep 64: 1-137.

8. Pastuszczak M, Gozdzialska A, Jakiela B, et al. (2017): Robust pro-inflammatory immune response is associated with serological cure in patients with syphilis: an observational study. Sex Transm Infect 93: 11-14.

9. Jung-In K, Ji-Hye P, Ju-Yeon C, et al. (2017): Serologic response to treatment in human immunodeficiency virus-nega- tive syphilis patients using automated serological tests: proposals for new guidelines. Ann Dermatol 29: 768-775.

10. Seńa AC, Zhang XH, Li T, et al. (2015): A systematic review of syphilis serological treatment outcomes in HIV-infected and HIV-uninfected persons: rethinking the significance of serological non-responsiveness and the serofast state after therapy. BMC Infect 15: 479-493.

11. Seńa AC, Wolff M, Martin DH, et al. (2011): Predictors of serological cure and serofast state after treatment in HIV-negative persons with early syphilis. Clin Infect 53: 1092-1099.

12. Zitti B, Bryceson YT (2018): Natural killer cells in inflammation and autoimmunity. Cytokine Growth Factor Rev 42: 37-46.

13. Qin J, Yang T, Wang H, et al. (2015): Potential predictors for serofast state after treatment among hiv-negative persons with syphilis in china: a systematic review and meta-analysis. Iran J Public Health 44: 155-169.

14. Huang J (2008): Detection of peripheral blood lymphocyte subsets in early sero-resistance syphilis. Chin J Derm Venereol 22: 546-547.

15. Tricco AC, Lillie E, Zarin W, et al. (2018): PRISMA extension for scoping reviews (PRISMA-ScR): checklist and explanation. Ann Intern Med 169: 467-473.

16. Ling X, Liu RQ, Shi X, et al. (2010): Determination of peripheral blood lymphocyte subsets in syphilitics of serofast and nerosyphilis and its significance. Chin J Derm Venereol 24: 418-420.

17. Xu G, Zheng JY, Mei SQ, et al. (2014): On patients with syphilis serum fixed detection of peripheral blood $\mathrm{T}$ lymphocyte subgroup interleukin-12, interleukin-10 research with the results of the level of NK cells. J Pract Med Tech 21: 1263-1265.

18. Liu Z, Chen JL, Liang XM, et al. (2015): Analysis of interleukin level in serofast syphilis patients. J Pract Dermatol 8: 102-106.

19. Li J, Wang LN, Zuo YG, et al. (2006): Clinical analysis and study of immunological function in syphilis patients with seroresistance. Natl Med J China 89: 813-816.

20. Xie HL, Zhao J, Li W, et al. (2013): The detection of cellular immunity of seroresistance syphilis patients. Chin J Derm Venereol 27: 49-51.

21. Liang FQ, Zhu XL, Peng JH (2016): Comparison of detection results of peripheral blood lymphocyte subsets with untreated syphilis. JiLin Med J 37: 2422-2423.

22. Yang RD, Cai CC, Tian GN, et al. (2005): Comparative study on peripheral blood lymphocyte subsets between untreated and serofast patients with syphilis. Chin J Dermato Venereol Integ Trad W Med 4: 15-17.

23. Guan Y, Yang F, Mo YS, et al. (2013): Clinical analysis of cellular immunity of 54 seroresistance syphilis patients. China Tropical Med 13: 1266-1268.

24. Xiong JH (2015): Clinical analysis and immune function study of syphilis serum resistance patients. Chin J Health Lab Tec 25: 88-89.

25. Bao TY, Yu NS, Zeng K (2006): Detection of the levels of IL-12, IL -10 in the serum among serofast patients with syphilis. Chin J Misdiagn 13: 254-255.

26. Zhang CY (2013): Clinical analysis of serofast patients among syphilitic patients. Lab Med Clin 10: 991-992.

27. Liu JL, Hu B, Zhao ZL, et al. (2010): Causes and correlative factors of serum-resistance of syphilis. Anhui Med Pharm J 14: 658-659. 
28. Huang Y, Cui XJ, Li SG, et al. (2018): Analysis of the expression of $\mathrm{T}$ lymphocyte subsets in the peripheral blood of patients with syphilis sero-fixation and its impact on the pathogenesis. Chin J Human Sex 27: 94-97.

29. Xie QP, Xing C, Zhang Q, et al. (2017): Analysis of peripheral blood lymphocyte subsets and its correlation with the rapid plasma reagin titers in serofast syphilis patients. Chin J Infect Dis 35: $165-168$.

30. Ruan LM, Liu XY, Jiang XL, et al. (2012): Serum-resistance of syphilis: an analysis of 66 cases. ZheJiang Med J 34: 786788.

31. Rouce RH (2015): More than memory: potential of adaptive natural killer cells. Biol Blood Marrow Transplant 21: 15341536.

32. Del ZG, Marcenaro E, Vacca P, et al. (2017): Markers and function of human NK cells in normal and pathological conditions. Cytometry B Clin Cytom 92: 100-114.

33. Vacca P, Munari E, Tumino N, et al. (2018): Human natural killer cells and other innate lymphoid cells in cancer: Friends or foes? Immunol Lett 201: 14-19.

34. Cruz AR, Ramirez LG, Zuluaga AV, et al. (2012): Immune evasion and recognition of the syphilis spirochete in blood and skin of secondary syphilis patients: two immunologically distinct compartments. PLoS Negl Trop Dis 6: e1717.

35. Geiger TL, Sun JC (2016): Development and maturation of natural killer cells. Curr Opin Immunol 39: 82-89.

36. Carlson JA, Dabiri G, Cribier B (2011): The immunopathobiology of syphilis: the manifestations and course of syphilis are determined by the level of delayed-type hypersensitivity. Am J Dermatopathol 33: 433-460.

37. Hawley KL, Cruz AR, Benjamin SJ, et al. (2017): IFN $\gamma$ enhances CD64-potentiated phagocytosis of treponema pallidum opsonized with human syphilitic serum by human macrophages. Front Immunol 8: 1227-1244. 